\title{
Von Zentralafrika nach Brasilien und Französisch- Guyana: Transnationale Migration, globale Ungleichheit und das Streben nach Hoffnung
}

\author{
Fabio Santos
}

\section{Einleitung}

Was in Zukunft passiert, was im Leben aus dir wird, nun, das ist ein trauriges Terrain, ehrlich, das ist Traurigkeit. Du denkst nach, du kämpfst und schlägst dich durch, aber du bewahrst immer Hoffnung ... Ich sage mir immer, dass sich eines Tages eine Gelegenheit ergeben wird und vielleicht können sich die Dinge ändern.

Célestin, November 2016

Wie gelangt jemand aus dem ärmsten Land der Welt, der Zentralafrikanischen Republik, nach Französisch-Guyana, einer südamerikanischen EU-Exklave, die nach globalem Maßstab einen hohen Lebensstandard aufweist? ${ }^{1}$ Diese Frage beschäftigte mich während meiner insgesamt sechsmonatigen Feldforschung in dem kaum bekannten französisch-brasilianischen Grenzgebiet und soll das vorliegende Kapitel

\footnotetext{
1 Obwohl Französisch-Guyana im Vergleich zur sogenannten Metropole - d.h. Frankreich ohne die sogenannten Überseegebiete - deutlich benachteiligt ist und beispielsweise eine signifikant höhere Arbeitslosigkeit aufweist (siehe z.B. Murdoch 2015: 70 ff.), ist das koloniale Überbleibsel nach globalem Maßstab wohlhabender als viele andere Staaten wie etwa die Zentralafrikanische Republik, die nach mehreren BIP-Berechnungen der Weltbank und des IWF das ärmste Land weltweit ist (Schieritz 2017). 
leiten, das sich an der Lebensgeschichte von Célestin orientiert und dabei Aufschlüsse über transnationale Migration im Kontext globaler Ungleichheitsrelationen gibt. ${ }^{2}$

Französisch-Guyana ist wie alle anderen „EU-Gebiete in äußerster Randlage“ (z.B. Mayotte und die Azoren) ein vollständiger Teil der Europäischen Union und bleibt dennoch in aller Regel unsichtbar in wissenschaftlichen und medialen Diskursen in und über Europa (Boatcă 2018, 2019). Dies ist umso erstaunlicher, wenn man bedenkt, dass es sich bei der durch den Amazonas-Regenwald verlaufenden Grenze zwischen dem nordbrasilianischen Bundesstaat Amapá und Französisch-Guyana um die längste Außengrenze Frankreichs handelt (Santos 2017). Obwohl die Migrationsbewegungen über diese Grenze sowie über die französisch-surinamische Grenze verstärkt in das Blickfeld vor allem ethnologischer Studien geraten sind, beschäftigen sich diese Studien fast ausschließlich mit Migrant*innen aus Brasilien, Surinam und Haiti (u.a. Arouck 2000; Mam Lam Fouck 2015; Martins/Superti/Pinto 2016; Piantoni 2011; Pinto 2016; Police 2010). So plausibel diese Schwerpunktsetzung aus quantitativen Gründen erscheint: Spätestens seit 2015 haben sich neue Migrationsmuster durch Europas „Hintertür“ in Südamerika herausgebildet, die auch die sogenannte „Europäische Flüchtlingskrise“ in ein neues Licht rücken (Santos im Druck).

In Anlehnung an sozialkonstruktivistische und figurationssoziologische Biografieforschung (Rosenthal 2016) waren quantitative Merkmale nicht ausschlaggebend für die Entscheidung, die Lebensgeschichte von Célestin zu analysieren und in den Kontext globaler Ungleichheiten einzubetten. Tatsächlich war Célestin der einzige aus dem sub-saharischen Afrika stammende Migrant, der mir im Grenzgebiet begegnet ist. Gerade in der Singularität jedoch liegt das Potential, auf gesellschaftlichen Wandel und größere Verflechtungszusammenhänge hinzuweisen. Dieser Ansatz hat sich bereits anhand der kolonial ähnlich gelagerten und dennoch deutlich präsenteren Grenzkonstellation zwischen Marokko und den spanischen Exklaven Ceuta und Melilla als erkenntnisweisend erwiesen (Rosenthal/Bahl/Worm 2017).

Das vorliegende Kapitel unternimmt den Versuch, die globalen Verflechtungszusammenhänge, in die Célestin qua Geburt eingebunden ist, unter Berücksichtigung seiner Agency und seiner Interpretation eben jener Zusammenhänge sowie seiner eigenen Lebensgeschichte zu analysieren. Die Verbindung dieser oftmals getrennt gehaltenen Analyseebenen erfordert neben der Interpretation unserer Gespräche ${ }^{3}$ die Einbettung in unterschiedlichste globale Szenarien, die von den Terroranschlägen vom 11. September 2001 bis zur globalen Finanzkrise reichen. Célestins Narrativ folgend, weist das Kapitel an manchen Stellen auch blinde Flecken auf, da einige Details nicht erinnert oder erzählt wurden. „Erinnern - das bedeutet für

2 Für alle angeführten Personen wurden Pseudonyme gewählt. Die genannte Frage war nicht leitend für das gesamte Forschungsprojekt, das sich mit der Konstitution verflochtener Räume im Grenzgebiet befasste. Célestin und ich haben uns über die NGO DPAC Fronteira kennengelernt, deren Mitarbeiter*innen zu wichtigen Bezugspersonen und Informant*innen während meiner Forschungsphase wurden.

${ }^{3}$ Neben zahlreichen informellen Alltagsgesprächen habe ich mit Célestin zwei lebensgeschichtlichnarrative Interviews in zumeist französischer Sprache geführt. Alle Zitate stammen aus diesen transkribierten und für das vorliegende Kapitel übersetzten Interviews. 
Migranten fast immer: Erinnerung an Verlorenes“, hat Arjun Appadurai (2016: 99) mit Blick auf die globalen Fluchtbewegungen des 21. Jahrhunderts geschrieben. Umso erstaunlicher sei das „Streben nach Hoffnung“ (ebd.), das diese teils lebensgefährlichen Bewegungen leitet. Auch Célestins Lebensgeschichte ist von einem solchen Streben gekennzeichnet, das seine Lebenssituation und damit auch seine Position im globalen Ungleichheitsgefüge nachhaltig verbessert hat. Nichtsdestotrotz sieht er sich auch heute in Französisch-Guyana mit zahlreichen Schwierigkeiten und Formen der Diskriminierung konfrontiert.

Nach der nun folgenden Einführung eines zentralen analytischen Rahmens zu global verflochtenen Ungleichheiten werde ich Célestins Lebensgeschichte in chronologischer Reihenfolge darstellen und analysieren: von Zentralafrika nach Kamerun, von Kamerun nach Spanien, von Spanien nach Brasilien, von Brasilien nach Französisch-Guyana.

\section{Global verflochtene Ungleichheiten}

Die soziologische Ungleichheitsforschung ist inzwischen verstärkt international und intersektional ausgerichtet. Diese Neuausrichtung besteht zum einen in der Überwindung des lange Zeit vorherrschenden methodologischen Nationalismus, zum anderen in der stärkeren Fokussierung auf die historisch konstituierte Verschränkung verschiedener Ungleichheitsachsen wie Staatsbürgerschaft, Geschlecht, Klasse und Ethnizität (Boatcă 2015; Jelin/Motta/Costa 2018; Weiß 2017). Eine Stärke dieses sich teils auf die Figurationssoziologie nach Elias stützenden Ansatzes (Braig/Costa/Göbel 2015) liegt in der Umgehung essentialistischer Kategorisierungen. Ob Kategorien wie die Konfessionszugehörigkeit, die sexuelle Orientierung oder der Bildungsgrad einer Person (mit-)entscheidend für die Position im Ungleichheitsgefüge sind, ist kontext- und somit auch ortsgebunden und kann nur am empirischen Fall geklärt werden.

Makroanalysen haben eindrucksvoll gezeigt, dass die in aller Regel zufällig erlangte und damit auch bei Geburt zugewiesene Staatsbürgerschaft einer Person ein zentraler - wenn nicht gar der zentrale - Faktor für die Position einer Person im globalen Ungleichheitsgefüge ist: „Pass und Visum gehören heute zu den wichtigsten Institutionen sozialer Ungleichheit.“ (Kreckel 2008: 26) Durch die Verschiebung der Analyseeinheit vom Nationalstaat hin zum Weltsystem haben Korzeniewicz und Moran diesen Zusammenhang detailreich analysiert und gegen verbreitete Annahmen wachsender Meritokratie argumentiert (Korzeniewicz/Moran 2009). Ihre Studie zeigt vielmehr, dass transnationale Migration sehr viel erfolgsversprechender für eine Person ist als beispielsweise die Erlangung von Bildung im nationalen Kontext, wie das folgende Beispiel verdeutlicht:

In Guatemala, for example, anyone belonging to the poorest seven deciles would be engaging in upward mobility by gaining access to the average income of the secondpoorest decile in Mexico. In Mexico, the incentives are even more striking, as all but the 
wealthiest decile would find upward mobility in gaining access to the average income of the second-poorest U.S. decile. (Ebd.: 108)

Die Autoren haben sich folgerichtig für die stärkere Berücksichtigung von Staatsbürgerschaft als zugeschriebenem Kriterium in der globalen Ungleichheitsanalyse ausgesprochen. Ein ähnliches Plädoyer wurde aus rechtswissenschaftlicher Sicht vorgelegt (Shachar 2009): Der Erwerb von Staatsbürgerschaft erfolge in der überwältigenden Mehrheit durch eine Art „Geburtsrechtlotterie“, nämlich den Zufall des Geburtsortes (im Falle von jus soli) oder der Abstammung (im Falle von jus sanguinis). In diesem oftmals als natürlich gegeben wahrgenommenen, tatsächlich aber regulierten System werden knappe Ressourcen durch das „Erbe“ einer bestimmten Staatsbürgerschaft beschränkt und Ungleichheiten auf globaler Ebene reproduziert.

In Bezug auf Französisch-Guyana lässt sich der von Korzeniewicz und Moran dargelegte Zusammenhang zwischen Migration und globaler Stratifikation zweifelsohne belegen. Durch den Status als französisches Übersee-Département und europäisches „Gebiet in äußerster Randlage“ ist Französisch-Guyana nach französischem und europäischem Maßstab zwar deutlich stärker von Arbeitslosigkeit, Armut und einem prekär ausgebauten Netzwerk an Gesundheits- und Bildungseinrichtungen geprägt. Global betrachtet und vor allem im Lichte der karibischen und südamerikanischen Nachbarschaft ist Französisch-Guyana jedoch ein attraktives Migrationsziel:

The benefits of decent schools, a superior system of public health, opportunities for menial jobs and generous social security benefits make this distant corner of Europe a beacon of hope for the immigrant Surinamers, Haitians, Brazilians, Chinese, Hmong and others who now (along with their Guyane-born children) make up well over half of the population. (Price 2018: 18)

Zudem wurde gezeigt, dass das jährliche Einkommen einer in Französisch-Guyana lebenden Person etwa fünfmal so hoch ist wie in Brasilien, zehnmal so hoch wie in Surinam und siebenunddreißigmal so hoch wie in Haiti (Marshall 2009: 221). Wie bereits erwähnt, handelt es sich bei den genannten Staaten um die drei Herkunftsländer der meisten in Französisch-Guyana lebenden Migrierten. Im Folgenden soll jedoch die Lebensgeschichte eines Mannes dargestellt werden, dessen Geburtsland in noch stärkerer Diskrepanz zu Französisch-Guyana steht: Laut Berechnungen des Internationalem Währungsfonds und der Weltbank weist die Zentralafrikanische Republik das geringste Bruttoinlandsprodukt der Welt auf. ${ }^{4}$

Neben der offenkundigen Rolle von Staatsbürgerschaft werden die oben aufgeführten, teils je nach Kontext unterschiedlich gewichteten Kriterien wie Ethnizität, Geschlecht und Religiosität in ihrem komplexen Zusammenspiel meine Analyse anleiten und die genannten Makroanalysen um eine in den globalen Kontext eingebettete mikrosoziologische Perspektive erweitern.

${ }_{4}$ Nachzuschauen unter https://data.worldbank.org/indicator/NY.GDP.PCAP.PP.CD?year_high_ desc $=$ true $<27.03 .2020>$ und https://www.imf.org/external/pubs/ft/weo/2018/01/weodata/index.aspx $<27.03 .2020>$. 


\section{Von Zentralafrika nach Kamerun}

Célestin wurde Ende der 1970er Jahre in politisch turbulenten Zeiten als viertes von sieben Kindern in Zentralafrika geboren, das zu der Zeit eine kurzlebige Monarchie (1976-1979) war und ab 1981 durch einen Staatsstreich zu einer Militärdiktatur unter der Führung von André Kolingba wurde. Aus Angst vor Repressionen beschlossen seine Eltern, das Land mit den Kindern in Richtung Kamerun zu verlassen, wo Célestins Eltern ursprünglich herstammen. Es kann davon ausgegangen werden, dass sein Vater (und durch ihn vermittelt auch seine Mutter, seine Geschwister und er selbst) über ein gewisses kulturelles Kapital verfügte, da er Célestin zufolge ein anerkannter Fotograf war, der bereits Jean-Bedel Bokassa ablichtete, den selbsternannten Kaiser des Zentralafrikanischen Reiches. Und dennoch: „Centrafrique ist immer im Kriegszustand, stets in Konflikten, auch heute noch“, sagte er in Bezug auf die Fluchtgründe der Familie. Den Rest seiner Kindheit und Jugend verbrachte er in dem frankophonen Teil Kameruns, wo seine Familie von Neuem beginnen musste. Da sein Vater Schwierigkeiten hatte, als Fotograf zu arbeiten, begann seine Mutter, den Lebensunterhalt der Familie als Putzkraft zu verdienen. Célestin sagte außerdem, dass sie ,gekocht hat, um das Essen auf der Straße zu verkaufen“. Im Rückblick auf seine Kindheit erklärte er diese schwierigen Bedingungen als zentralen Anstoß für seinen Fleiß in der Schule, die er 1999 in Bafoussam, der Hauptstadt der westlichen Region Kameruns, abgeschlossen hat:

Ich wurde sehr hart erzogen, wirklich, wir haben nie meinen Geburtstag gefeiert, niemals, so wurde ich erzogen aufgrund der Armut ... Und ich musste kämpfen, um zur Schule zu gehen, um meinen Abschluss zu machen ... Ich selbst musste dafür kämpfen, meine Bücher zu bekommen: In den Ferien habe ich immer nach etwas zu tun gesucht, und mit diesem Geld habe ich dann meine Schulbücher gekauft. Mein Leben war wirklich ... immer sehr hart, nichts ist einfach, ich musste immer enorme Anstrengungen unternehmen, um etwas zu erreichen, um die Universität zu besuchen, es gab überall Hindernisse, ich musste mich enorm anstrengen ... um diese Hindernisse zu überwinden. So ist das Leben.

Nach dem Sekundarschulabschluss nahm Célestin ein naturwissenschaftliches Studium in Kameruns Hauptstadt Yaoundé auf. Allerdings sei ihm schon sehr früh klar gewesen, dass er ins Ausland gehen wollte. Obwohl sein Studium seiner Darstellung nach reibungslos verlief, spürte er eine Perspektivlosigkeit, die als „social death among those who can neither find work nor move“ (Andersson 2014: 19) bezeichnet wurde. Er wusste, so teilte er mir rückblickend mit, dass er in Kamerun selbst mit einem Hochschulabschluss kaum eine feste Arbeit und ein auskömmliches Einkommen finden würde. Diese Einschätzung deckt sich mit wissenschaftlichen Studien, die einen „routinized state of crisis“ (Alpes 2017: 306) in Kamerun diagnostiziert haben, der wiederum eng mit Staaten und Institutionen des globalen Nordens verwoben ist: „Migration aspirations have become an essential part of society in Cameroon in the wake of the economic crisis following the structural adjustment 
programmes of the International Monetary Fund and the devaluation of the country's currency, the CFA franc." (Ebd.)

In Anbetracht dieser strukturell bedingten Aussichtslosigkeit erscheint es kaum verwunderlich, dass Célestin früh einen alternativen Lebensweg plante. Sein erklärtes Ziel war es, sein Studium in einem anderen Land fortzusetzen, vorzugsweise in Deutschland, Italien, Kanada oder den USA. Deutschland war für ihn ein naheliegendes Ziel, da er bereits auf dem Gymnasium Deutsch gelernt hatte. ${ }^{5}$ Dieser Plan wurde jedoch von den strikten Auflagen durchkreuzt, die vorsahen, dass afrikanische Studierende in Deutschland einen Lebensunterhaltsnachweis von rund 10.000 Euro vorweisen mussten. „Das ist nur für Familien, die viel Geld haben“, winkte er mir gegenüber nachträglich ab. Italien hingegen erschien Célestin als durchaus ,,erschwinglich“: So waren in Italien „nur“ 4.000 Euro vorzuweisen. Da ihm das Ansparen dieser Summe in Kombination mit Leihgaben von Freund*innen und Familienmitgliedern als realistisch erschien, nahm er an einem einjährigen Italienischkurs in Yaoundé teil. Allerdings bestand er die Abschlussprüfung dieses Kurses nicht, weshalb seine Migration nach Italien vorerst auf Eis gelegt wurde. ${ }^{6}$

Parallel zu seinen Italienplänen eruierte Célestin zwei weitere Möglichkeiten: Zum einen beantragte er im Rahmen des Diversity-Visa-Programms (der sog. Green-Card-Lotterie) eine Aufenthalts- und Arbeitsgenehmigung für die Vereinigten Staaten. Zum anderen bemühte sich einer seiner Professor*innen, ihn im Rahmen von Austauschprogrammen an eine kanadische Universität zu vermitteln. Die Resultate dieser Optionen hat er wie folgt beschrieben:

All dies geschah 2001, und ich habe völlig unerwartet die Green Card erhalten. Aber zu dem Zeitpunkt gab es die Angriffe auf das World Trade Center, und die amerikanische Regierung hat uns erklärt, dass sie die Zahl der Visa reduzieren wird. Ich habe den Brief bereits in meinen Händen gehalten, mit Glückwünschen und allem, ich wurde angenommen, aber nach allem, was mit dem World Trade Center passiert ist, wollten sie die Zahl der Visa reduzieren, und das hat mich wirklich erschüttert. Ich wollte doch unbedingt mein Studium im Ausland fortsetzen! In der Zwischenzeit versprach mir mein Professor ein Stipendium in Kanada, das war meine letzte Gelegenheit, aber daraus wurde nichts. An diesem Punkt bin ich gegangen, einfach so, ohne meiner Familie etwas zu sagen, ich war total enttäuscht, also habe ich meinen Rucksack gepackt und bin abgehauen.

\footnotetext{
${ }^{5}$ Im Jahr 2003, als Célestin Kamerun verlassen hat, waren circa 5.300 kamerunische Studierende an deutschen Universitäten eingeschrieben (Mberu/Pongou 2016).

${ }^{6}$ Die genauen Gründe für das Nichtbestehen Célestins, der sich mir gegenüber stets als fleißig und klug präsentierte und - später in Brasilien - zwischenzeitlich selbst als Lehrer arbeitete, sind in unseren Gesprächen unklar geblieben. Mir scheint eine Mehrfachbelastung durch Arbeit, Studium, Sprachkurs und familiäre Verpflichtungen jedoch die naheliegende Erklärung zu sein. Tatsächlich klaffen die Lebensgeschichten von Célestin und seinem an dieser Stelle aus Platzgründen nicht weiter eingeführten jüngeren Bruder ab diesem Punkt auseinander. Sein Bruder, der sich Célestin als Vorbild nahm und den Kurs später auch besuchte, bestand den Test und erhielt einen Studienplatz in Bologna. Heute arbeitet er als Apotheker in Hamburg. Auch diese eindrucksvolle Migrationsgeschichte deutet auf ein Mindestmaß an kulturellem Kapital in der Familie hin, das zumindest teilweise in ökonomisches Kapital umgewandelt werden konnte.
} 
Nachdem seine Green Card annulliert wurde und somit keiner seiner Migrationspläne wie erhofft aufging, ist Célestin nach eigener Aussage auf eigene Faust gegangen, auch wenn Freund*innen und Verwandte in den grundsätzlichen Plan zu migrieren eingeweiht waren. Er sah, so wie er es formulierte, keine andere Möglichkeit, als ,,illegal zu werden“: „Für den größten Teil meines Abenteuers war ich ein illegaler Einwanderer, einer von denen, die niemand will, für die sich niemand interessiert." Mir scheint, dass Célestin den Begriff ,illegaler Einwanderer“ mit Bezug auf die Fremdbeschreibung durch andere verwendete. Anders ausgedrückt benutzte er hiermit einen weitverbreiteten und oftmals unhinterfragten Begriff im Bewusstsein, dass er selbst während seines noch zu erläuternden Migrationsweges nie ,illegal“ im engeren Wortsinn war, denn dieser Begriff ist „pejorative, stigmatizing, and even incorrect, implying as it does that migrants are criminals when they have usually only committed an administrative infraction" (Andersson 2014: 17).

Als Célestin, der ein klarer Verlierer der „Geburtsrechtlotterie“ ist, seine Position mittels transnationaler Migration zu verbessern suchte, erkannte er ,the harsh reality ... that not everyone who wishes to leave his or her country of origin will be able to lawfully enter a desired destination country" (Shachar 2014: 114). Tatsächlich war sein Ziel mit Händen zu fassen und er wurde zu einem vorübergehenden Gewinner der Green-Card-Lotterie. Dieser Gewinn war jedoch von kurzer Dauer, da die US-amerikanische Regierung aufgrund der Anschläge vom 11. September eine Reihe von erteilten Aufenthaltsgenehmigungen zurückzog. Das machte Célestin gewissermaßen zu einem doppelten Verlierer zweier Staatsbürgerschafts- und Wohnsitzlotterien. Zudem wurde er nicht nur in einem Land geboren, das ihm weitaus weniger Mobilitätsmöglichkeiten bot als Staatsbürgerschaften des globalen Nordens, sondern er betonte auch, dass er einfachen Verhältnissen entstammt, auch wenn er anscheinend über ein für kamerunische Verhältnisse überdurchschnittliches kulturelles Kapital verfügte. Dennoch zeigt sich, dass die Verschränkung von Staatsbürgerschaft und Klasse (hier vor allem im ökonomischen Sinne) eine „legale“ Migration bedeutend einschränkte. So war Célestin beispielsweise nicht in der Lage, sich in legale Mobilitätsprogramme eines seiner priorisierten Ziele (Deutschland) und die notwendige sprachliche Vorbereitung „einzukaufen“. So sah er sich gezwungen, sich auf einen beschwerlichen, illegalisierten Weg in Richtung Europa zu begeben.

\section{Von Kamerun nach Spanien ${ }^{7}$}

Célestins erklärtes und naheliegendes Ziel war Italien, da er bereits einen Italienischkurs absolviert hatte. Der einzig mögliche Weg für ihn führte durch die Sahara und

\footnotetext{
${ }^{7}$ Es lohnt sich, eine parallele Lektüre des vorliegenden Kapitels mit der Lebensgeschichte des anglophonen Kameruners Franklin vorzunehmen, der sich etwa zehn Jahre nach Célestin auf den Weg nach Melilla machte. Trotz einiger Unterschiede wie der schnelleren Migration Franklins lassen sich ähnliche Muster wie die Mobilisierung kulturellen Kapitals und der Wunsch nach Bildung festmachen (Rosenthal/Bahl/Worm 2017: 128 ff.).
} 
anschließend über das Mittelmeer. Zu Beginn reiste er von Douala in Kamerun nach Calabar und Kano in Nigeria und von dort in den Niger. Célestin schien keine ernsthaften Probleme beim Überschreiten dieser Grenzen zu haben. Von Agadez im Niger aus verfolgte er die sogenannte „northern route“ in Richtung Libyen. Zum Zeitpunkt seiner Migration Anfang der 2000er Jahre war dies mit 60.000 bis $80.000 \mathrm{Mig}$ rierenden jährlich die wichtigste Route von Afrika nach Europa (Simon 2006: 54).

Célestin hat mir gesagt, dass ein Großteil dieser Route in größeren Geländewagen mithilfe von sogenannten „Schmuggler*innen“ durchgeführt wurde, was jüngsten Forschungsergebnissen entspricht, die darauf hinweisen, dass diese Route ohne die Bestechung von Grenzbeamt*innen und die Inanspruchnahme von „Schmuggler*innen" kaum möglich ist (Crawley et al. 2017: 35). Anstatt diese Praktiken einseitig aus einer staatszentriert-konservativen Perspektive als ,illegal“ zu verurteilen, können sie aber auch als Gegenstrategien in einem global ungleich verflochtenen Weltsystem verstanden werden: „The migration facilitation services offer real avenues of hope for geographical mobility. Aspiring migrants relate the potential trickery of a broker to the institutionalized sabotage of their migration ambitions, notably by destination states that deny visas.“ (Alpes 2017: 308)

Um die algerische Oasenstadt Djanet im Dreiländereck von Algerien, Niger und Libyen zu erreichen, musste er sich mit einem Geländewagen durch die Wüste fahren lassen. Diesen Weg beschrieb er wie folgt: „Wenn du Glück hast, lässt die Polizei dich weiterfahren. So war es bei mir. Mein Weg nach Djanet dauerte eine Woche, aber andere sind gestorben. "Célestin berichtete mir mit leiser Stimme, fast flüsternd, von dem Tod so vieler anderer, die ähnliche Migrationsziele wie er verfolgten. Von Djanet aus reiste er in die libysche Grenzstadt Ghât, was als ,most dangerous and deadly land route that migrants can take" (Simon 2006: 54) beschrieben wurde. Sein Leid, die Verzweiflung und sein Überleben brachte Célestin im weiteren Verlauf des Gesprächs zum Ausdruck:

In der Wüste ... das ist unglaublich, es war sehr ... am Ende habe ich es geschafft, aber manchmal wusste ich nicht, ob ich stark genug bin. Ehrlich, es gibt Leute, die das nicht ausgehalten haben. Das ist die Wüste und es gibt kein Wasser. Und so ist es mir ergangen: Das Wasser war aus, deshalb habe ich meinen eigenen Urin getrunken, weil es kein Wasser gab und du nicht weißt, wann du wieder etwas zu trinken bekommst. ... Wir haben einen Tuareg gesehen, es war ein Tuareg, der uns gerettet hat. Wir haben viele gesehen, die gestorben waren. Keine Ahnung, das Schicksal wollte, dass ich ankomme.

Diese verstörende Geschichte ist jedoch keine Ausnahme, denn „refugees and migrants described the long desert crossing in treacherous conditions which often ends in starvation, thirst, and sometimes death" (Hamood 2008: 31). Eine aktuellere Studie über Fluchtbewegungen kam zu dem Schluss, dass jede fünfte Person, die auf ihrem Weg nach Europa die Sahara durchquerte, Leichen sah oder bei dem Tod anderer anwesend war (Crawley et al. 2017: 77).

Zusätzlich zu dieser akuten Lebensbedrohung sah sich Célestin nach seiner Ankunft in Libyen mit zahlreichen weiteren Problemen konfrontiert. „Zu diesem Zeitpunkt war es Muammar al-Gaddafi, der alles kontrollierte, es war hart", sagte er mir. 
Kein anderes Land habe er als so außerordentlich feindlich gegenüber Menschen auf der Flucht empfunden, regelmäßig habe er von Verhaftungen von Migrant*innen gehört oder diese selbst gesehen. Dies lässt sich wiederum auf die Anti-Immigrationspropaganda des damaligen Regimes zurückführen: 2003 wurden circa 43.000 Menschen ausgewiesen, 2004 waren es 54.000 (Simon 2006: 32f.). In wohl kaum einem anderen Land ist zudem die ethnische Diskriminierung so stark wie in Libyen, wo Schwarze Migrant*innen häufig zu Opfern polizeilicher Despotie und neuen Formen von Sklaverei werden (Crawley et al. 2017: 98 ff.), auch wenn dies eine Entwicklung der letzten Jahrzehnte ist, wie an anderer Stelle dieses Sammelbandes aufgezeigt wird (s. Beitrag von Steve Tonah). Unter diesen Bedingungen verschwimmt auch die vermeintlich klare Trennlinie zwischen „erzwungener" und „freiwilliger“ Migration: „Even though the initial decision to migrate may have been primarily driven by economic factors, many ... decided to leave Libya due to factors that are more typically associated with forced migration." (Crawley et al. 2017: 99)8 Im Falle Libyens kommt hinzu, dass es nach damals geltendem Recht und offizieller Rhetorik überhaupt keine Geflüchteten auf libyschem Territorium geben durfte (Hamood 2008: 25).

Wie also ging Célestin mit diesem von ihm als höchst gefährlich eingeschätzten Kontext um? Obwohl Célestin, wie er sagte, im Alltag oftmals aufgrund seiner Hautfarbe als Migrant „enttarnt“ wurde, hat er eine Strategie entwickelt, die ihn vor weiteren verwobenen, sich gegenseitig verstärkenden Diskriminierungen schützte, allen voran mit Blick auf seine christliche Konfession. So hat er hat mir erzählt, wie er sich im libyschen Alltag oftmals als Muslim ausgab, um Hilfe zu erhalten und in die Moschee zu gehen: „Ich lernte das muslimische Gebet, nicht alles, nur den Anfang und das Ende. Manchmal ging ich in die Moschee, es hat immer funktioniert. Ich lernte, wie man die Kleidung richtig trägt, wie man das Gebet macht.“ Seiner Erzählung zufolge entlastete diese Strategie seinen Alltag in Libyen und weiteren muslimisch geprägten Ländern. Dennoch wollte er Libyen schnellstmöglich verlassen. Der Weg über das Mittelmeer nach Italien erschien ihm jedoch zu riskant. Deshalb überlegte er nach anderen Möglichkeiten: „Du denkst dir: Welchen Weg werde ich nehmen, wie wird es weitergehen? Du hast Zweifel: Wie kann ich aus dieser Situation herauskommen?"

Schließlich beschloss Célestin, es über die spanische Exklave Melilla im Nordosten Marokkos zu probieren. Um auf diesem Weg auf spanisches und somit EUTerritorium zu gelangen, musste er jedoch drei weitere Grenzen überqueren: die libysch-algerische, die algerisch-marokkanische und schließlich die von diesen mit Abstand am stärksten überwachte Grenze zwischen Marokko und Spanien (Melilla). Er erzählte mir, dass er bei Ghadames die libysch-algerische Grenze überquerte und

\footnotetext{
8 Ich habe mich entschieden, Célestin als Migranten zu bezeichnen, da ich diese Bezeichnung als Oberbegriff verstehe, der verschiedene Beweggründe und Dringlichkeiten zu verschiedenen Zeitpunkten und an verschiedenen Orten einbezieht. Die Perpetuierung des vermeintlichen Gegensatzpaares (freiwillige ${ }^{\mathrm{r}}$ ) Migrant*in vs. Geflüchtete*r führt allzu oft zu dem Ausschluss von Menschen von dem internationalen Schutz, den sie eigentlich benötigen (s. auch Crawley et al. 2017: 8).
} 
dann eine schwierige Reise durch Algerien unternahm, die er aber durchstand, da er sich sagte: „Ich hatte jetzt ein klares Ziel. Das war meine letzte Chance.“ Seine Erfahrungen in Algerien, über die er in unseren Gesprächen nicht viel erzählte, scheinen überdeckt von den Erfahrungen im spanisch-marokkanischen Grenzgebiet: Monatelang harrte er auf dem marokkanischen Berg Gourougou aus, der seit Anfang der 2000er Jahre das temporäre Zuhause für Tausende Migrant*innen und zugleich für einige von ihnen das fast buchstäbliche „Sprungbrett“ in die EU wurde. ${ }^{9}$ Von hier aus unternahm er mehrfach den Versuch, über die zwei Stacheldrahtzäune zu „springen“ (frz. „sauter"), die damals je drei Meter hoch waren:10

Man musste über die Barriere springen. Ich habe es siebenmal versucht. Nur sehr wenige Menschen schaffen es beim ersten Mal. Manchmal, bei Nacht, hat man Glück. Wenn nicht, gehst du zurück nach Gourougou, schläfst und lässt dabei deine Schuhe an, weil die marokkanische Gendarmerie jederzeit kommen kann. Sie kamen viele Male, als ich dort im Lager lebte. Sie verfolgen dich, sie verbrennen deine Sachen, es ist verrückt. Und wenn du zur Grenze oder hinunter nach Beni Ensar gehst, halten sie dich an, sie schlagen dich, sie verhaften dich, sie stecken dich ins Gefängnis oder bringen dich zurück. Aber wir, da es unser Ziel war, nach Spanien zu gehen, kamen wir immer wieder zurück. Es ist wie ein Spiel. Das war wirklich die härteste Grenze. Ich habe einen Freund, der sein Auge verloren hat, ein anderer hat sich das Bein gebrochen, die Zäune haben Stacheldraht und sind drei oder vier Meter hoch, man muss den ersten Zaun hochklettern und dann zum zweiten springen, weil sie zwei Zäune haben. Also, als einer von den anderen sprang, hat er sich das Bein gebrochen. Der andere ist bis heute blind. Ich habe sieben Versuche gemacht und auch kleine Verletzungen erlitten. Am 7. März 2005, es war nachts, da versuchte ich es noch einmal und wir hatten eine Leiter und dann sprang ich, ich sprang, ich sprang und ich habe es geschafft. Da war ich, in Spanien, aber es gab auch die spanische Polizei, die schoss mit Gummigeschossen und rannte hinter uns her, einige haben es geschafft, andere nicht. Wenn du also ankommst, musst du dich verstecken, du musst ein Versteck finden, denn sonst werden sie dich mitnehmen, die spanische Gendarmerie wird dich festnehmen und zurückschicken. Du musst bis zum Sonnenaufgang warten und dann ins Flüchtlingszentrum gehen. Nun, ich hatte Glück, ich konnte mich vor der Polizei verstecken, und dann bin ich am Morgen zum Zentrum gegangen.

519 Tage nach seiner Abreise aus Yaoundé erreichte Célestin endlich sein Ziel: Obwohl er nicht, wie ursprünglich geplant, in Italien war, so war er doch in der EU. Noch heute betrachtet er seine Ankunft als Schlüsselmoment für den Verlauf seines weiteren Lebens: „Erst als ich in der Ankunftsstelle war, kam ich richtig an. Ich wurde neu geboren." Doch er hat mir auch von körperlichen wie psychischen Fol-

\footnotetext{
${ }_{9}^{9}$ Der Dokumentarfilm Les sauteurs (2016) gibt interessante Einblicke in das Alltagsleben auf dem Berg Gourougou aus der Perspektive eines Migranten.

10 2005, kurz nach Célestins Ankunft in Melilla, wurde ein dritter Zaun errichtet und die Höhe aller Zäune wurde verdoppelt. Diese Maßnahme kann als Teil des von der EU initiierten „Global Approach to Migration and Mobility" (European Commission 2011) verstanden werden. In diesem Zusammenhang ist es wichtig zu erwähnen, dass die „Sicherung“ der Grenzen zwischen Spanien und Marokko eng mit dem EU-Beitritt Spaniens 1986 verflochten ist (Bahl 2017: 185).
} 
gen seiner Migrationsroute berichtet. Seine knapp anderthalb Jahre währende Route bis dorthin glich eher einem „Überleben, nicht Leben“, wie er sagte:

Fabio, ich habe niemanden. Viele von uns haben Kopfschmerzen, die verschwinden nicht. Es ist eine Menge. So ist das Leben, das Leben geht weiter, aber es gibt viele Dinge, die wir, tief im Inneren ... weißt du ... ich weiß nicht, wie ich es erklären soll. Es ist viel Leid. Wenn du ankommst, gibt es niemanden, keinen Psychologen, der dir hilft, wenigstens ein bisschen. Und wenn du dort ankommst, beginnt das Abenteuer von Neuem. Wenn du ankommst, denkst du, alles ist vorbei, aber nein, ein neues Abenteuer beginnt. Du hast kein Geld, du hast keine Papiere, du hast nichts, du kannst nicht arbeiten. Also beginnt ein weiteres Abenteuer.

\section{Von Spanien nach Brasilien}

Sechs Monate verbrachte Célestin im Centro de Estancia Temporal de Inmigrantes in Melilla. Wie Tausende andere Migrant*innen war er einer „politics of time“ unterworfen, die typisch für Ceuta und Melilla ist und als eine Art Abschreckungsmaßnahme gegen weitere Grenzübertritte gedeutet werden kann (Andersson 2014: 24). Zwar lernte er in Melilla Spanisch, doch hauptsächlich warteten er und die anderen Migrant*innen tagein tagaus darauf, in eine der anderen spanischen Regionen ,,verlegt“ zu werden. Célestin hatte kein Mitspracherecht, wohin er „,versetzt“ werden könnte, und so brachte die administrative „Lotterie“ ihn schließlich nach Madrid.

Im Madrider Barrio Aluche musste Célestin einen Monat im lokalen Centro de Internamiento de Extranjeros verbringen, einem Ausweisungszentrum, in dem Zugewanderte bis zu zwei Monate lang festgehalten werden. Allerdings werden 71 Prozent der Inhaftierten nicht ausgewiesen. ${ }^{11}$ Auch Célestin wurde nach mehreren Wochen entlassen und versuchte in dieser für ihn völlig neuen Umgebung, ,ein normales Leben“ zu führen, wie er sagte: Freundschaften schließen, einen Job und eine Wohnung finden. Doch ohne Kontakte und finanzielle Ressourcen befand sich Célestin schon bald am Rande der spanischen Gesellschaft:

Nun, ich, ich kannte niemanden. Ich habe angefangen, unter einer Brücke zu schlafen, da in Madrid gibt es diese Brücke [Brücke von Segovia; Anm. des Autors] in der Nähe der Kathedrale [Almudena; Anm. des Autors] und des Palastes. Die Leute schlafen dort, weil sie nirgendwo sonst hingehen können. Ich bin in Madrid nicht vorangekommen, man ist darauf angewiesen, einige kleine Arbeiten zu erledigen, ich habe auf Autos aufgepasst. Du passt auf sie auf und die Leute geben dir ein, zwei Euro, wenn sie wiederkommen, das ist allein zum Überleben. Danach bin ich nach Santander gegangen.

Célestin hat erzählt, dass er in Madrid von einem Bekannten hörte, dass es besser sei, die spanische Hauptstadt zu verlassen. Die Stadt sei zu voll und es gebe kaum Möglichkeiten für Migrant*innen. Während seiner ersten drei Monate in Santander kam er in einem Kloster und bei einem Verein unter, der Migrant*innen beherbergt.

$11 \mathrm{Zu}$ dem Thema hat die spanische Tageszeitung El País eine gut recherchierte Online-Sonderausgabe herausgegeben (El País 2017). 
Erst in Santander erhielt er eine tarjeta de residencia, die ihm viele Aktivitäten ermöglichte - von der Eröffnung eines Bankkontos bis zur Wahrnehmung von Bildungsangeboten. Während dieser Zeit hielt er sich über Wasser, indem er auf Müllplätzen und in Containern nach Autorädern gesucht hat. Dies war, wie er mir sagte, „extrem harte Arbeit, eine anstrengende Arbeit", die er nicht längerfristig verfolgen konnte und wollte. Zu diesem Zeitpunkt hatte sich sein Spanisch bereits deutlich verbessert und er war aktiv auf der Suche nach Fortbildungen. In Santander absolvierte er mehrere kürzere Ausbildungsprogramme, deren Zertifikate er mir stolz in der Pension zeigte, in der er während meines Forschungsaufenthaltes 2016 im nordbrasilianischen Oiapoque lebte: Sie alle bildeten Célestin in praktisch-handwerklichen Bereichen fort: Industriereinigung, Schweißerei und Hafenarbeit. Einige dieser Ausbildungsprogramme wurden von Fördermitteln der Europäischen Union finanziert, deren Logo auf den Zertifikaten blau und gelb glänzte. Obwohl Célestins Fortbildungsanstrengungen langfristig nicht von Erfolg gekrönt waren, hat er mir noch Jahre später mit einer Mischung aus Stolz und Bescheidenheit von seinen ersten Erfolgsgeschichten in Spanien erzählt:

Die Ausbildung zum Schweißer, das war eine acht Monate dauernde Ausbildung. Es war ein hartes Auswahlverfahren, es gab 150 Bewerber, aber sie wollten nur 15 Personen, und ich wurde als eine von diesen 15 Personen ausgewählt, weil sie einen Spanischtest gemacht haben. So wurde ich ausgewählt.

Jedoch führte keine der Fortbildungen zu einer ernsthaften beruflichen Perspektive in Zeiten der Finanzkrise. Von Zeit zu Zeit verdiente er sich unter der Hand etwas Geld hinzu und erhielt zudem eine monatliche finanzielle Unterstützung aus den genannten Fortbildungsprogrammen. So gelang es Célestin, der rigoros an allen Ecken und Enden sparte, im Laufe der Jahre etwas Geld beiseitezulegen, obwohl er zusätzlich Geld an seine Familie in Kamerun schickte. Doch in den Jahren 2012 bis 2013, so sagte er mir, kam er in große Schwierigkeiten. Wie eine Vielzahl von Menschen in Spanien und der ganzen Welt war er ganz persönlich von der globalen Finanzkrise betroffen. Auf dem Höhepunkt der Krise war er noch immer in Santander und fand keine Beschäftigung, sei diese informeller oder formeller Natur. Deshalb beschloss Célestin, umzuziehen:

Ich bin nach Barcelona gezogen. In Barcelona, dachte ich, hätte ich mehr Möglichkeiten. Ich lernte Katalanisch und es gab die Option, als Taxifahrer zu arbeiten. Ich hatte meinen Führerschein in Santander erhalten, und so habe ich mit einem Kurs zum Taxifahrer angefangen. Aber während des Kurses habe ich gemerkt, dass die Bedingungen es mir nicht erlaubten, einen ordentlichen Führerschein als Taxifahrer zu erwerben. Inzwischen verschlimmerte sich die Situation, ich fand nichts, und an dieser Stelle lernte ich eine Dame aus Brasilien [Marcia, die seine Partnerin wurde; Anm. des Autors] und einen Brasilianer kennen, der mir sagte, dass es in Manaus viele Möglichkeiten für Schweißer gebe. Er hat mir gesagt, dass er mir helfen könne, eine Arbeit in einer Firma in Manaus zu finden. Und zufällig hatte sie [Marcia; Anm. des Autors] in Manaus gelebt, und so wollten wir dorthin gehen, auf diese Weise bin ich nach Brasilien gekommen. 
Célestin und seine brasilianische Partnerin Marcia haben all ihre Ersparnisse zusammengetragen und 2013 einen Flug nach Manaus gebucht. Der Mangel an Perspektiven inmitten der Weltfinanzkrise, von der Spanien in besonderem Maße betroffen war, veranlasste sie, Europa zu verlassen. Anders als viele europäische Länder erlebte Brasilien seit Anfang der 2000er Jahre einen starken wirtschaftlichen Aufschwung, der gewissermaßen auch vor der globalen Finanzkrise schützte: Das brasilianische Wirtschaftswachstum ,was followed by a sharp reduction in unemployment rate, from 12.4\% in 2003 to 5.1\% in 2013“ (Prates/Fritz/Paula 2017: 20). In Spanien hingegen lag die Arbeitslosenquote zu dieser Zeit bei über 25 Prozent. In dieser Phase des globalen und für ihn auch persönlichen Umbruchs kultivierte Célestin das von Appadurai beschriebene und viele Migrant*innen weltweit kennzeichnende Streben nach Hoffnung und hoffte auf eine bessere Zukunft in Brasilien.

\section{Von Brasilien nach Französisch-Guyana}

Als Célestin und Marcia in Manaus, der Hauptstadt des brasilianischen Bundesstaates Amazonas, ankamen, gab es keine Spur von Célestins Bekanntem, der ihm eine Anstellung in Aussicht gestellt hatte. Er versuchte, das Beste aus seiner Situation zu machen und bewarb sich auf verschiedene Stellen, erneut ohne Erfolg. Immerhin fand er nun im Privaten eine gewisse Stabilität, die sich wiederum auf seinen Aufenthaltsstatus in Brasilien auswirkte: Marcia und Célestin sind in Brasilien eine eingetragene Lebenspartnerschaft eingegangen, die Célestin eine langfristige Perspektive in Brasilien bot. Neben kleineren Hilfstätigkeiten bot sich ihm und Marcia jedoch keine Perspektive. Deshalb entschlossen sich die beiden zu einer Neuorientierung: Während Marcia zunächst in Manaus bleiben würde, sollte Célestin die Möglichkeiten eines Umzugs nach Französisch-Guyana ausloten. Erst in Brasilien wurde ihm diese „Hintertür“ der EU in Südamerika bewusst. Mit dem Bus reiste er 2014 nach Boavista, von dort aus nach Georgetown in Guyana und anschließend über Surinam nach Französisch-Guyana. Wie gestaltete sich seine Situation vor Ort?

Ich bin dort angekommen, aber es war nicht so, wie ich es mir erhofft hatte. Ich habe ihnen meine Situation erklärt, meine Dokumente gezeigt und dass ich legal in Europa gewohnt habe, dass ich eine Ausbildung habe, dass ich Ausbildungen gemacht habe, die von europäischen Geldern finanziert wurden. Sie haben gesagt, nein, es gibt hier keine Lösung für dich, du musst zurück nach Spanien gehen. Ich habe dort sogar Asyl beantragt, aber das hat nicht funktioniert.

Célestin hat versucht, in dem französischen Übersee-Département Fuß zu fassen. Zwar fand er von Zeit zu Zeit kleinere sporadische Hilfstätigkeiten, doch diese ähnelten teilweise ausbeuterischen Verhältnissen. So berichtete er mir von einer Situation, in der er einem Mann bei dem Ausbau seines Hauses geholfen hat, ohne im Anschluss das versprochene Geld zu erhalten. „Cheap and willing immigrant labour is everywhere in Guyane“ (Price 2018: 22), doch wird dieses „Überangebot“ an billiger Arbeitskraft häufig zulasten der sich in prekären Lebenssituationen befindlich- 
en Migrant*innen ausgenutzt. Nach mehreren Monaten, in denen er in einer bescheidenen Unterkunft in einem der vielen, vornehmlich von Migrant*innen bewohnten Außenbezirke Cayennes wohnte, erhielt er einen negativen Asylbescheid vom Cour Nationale du Droit d'Asile (CNDA). Marcia und er beschlossen, sich in Oiapoque zu treffen, der nordbrasilianischen Stadt, die direkt an Französisch-Guyana grenzt und Schauplatz komplexer Aushandlungen von Zugehörigkeit und Grenzüberschreitung war und ist (Santos 2020).

In Oiapoque lebten die beiden vorübergehend in einer für diese Region typischen, aus bunt gestrichenem Holz gebauten Pension, in der sie die Miete durch Marcias Tätigkeit als Putzkraft ausglichen. Célestin engagierte sich in dieser Zeit als Freiwilliger in der NGO DPAC Fronteira und gab dort Französischkurse. In diesem Zusammenhang wurden wir einander von Mitarbeitenden der Organisation vorgestellt. Wir lernten uns daher an einem wichtigen Scheideweg seiner Migrationsgeschichte kennen: Zu dieser Zeit in Oiapoque beschloss er schließlich, weiterhin nach Hoffnung zu streben und Berufung gegen das Urteil des CNDA einzulegen. Angesprochen auf seinen größten Wunsch und sein Ziel, sagte er mir zum Zeitpunkt seines zweiten Asylantrags:

Mein Traum, ganz ehrlich, ist es zu studieren. Ich bin noch nicht fertig. Man sagt, ein Mensch ohne Bildung ist kein richtiger Mensch. Ich habe das immer gewollt, eine richtige Ausbildung zu machen, eine richtige Ausbildung, um danach zu arbeiten. Alle Ausbildungen, die ich da gemacht habe, wie Schweißerei und all das, ehrlich, ich habe so hart dafür gekämpft, um das in Spanien zu haben. Das hat mich so deprimiert, so deprimiert. Wenn sie mir Asyl in Guyane gewähren, werde ich mich an der Universität einschreiben, denn ich kann das tun mit meinem Abitur. ... Ich will wirklich an die Universität zurückkehren, selbst wenn ich mittlerweile verstanden habe, dass das Schicksal mir das vielleicht nicht erlauben wird. Manchmal sind es nur zwei Jahre, du kannst Telekommunikation in zwei Jahren studieren. Mein Traum war es, das in Deutschland zu machen, dort gibt es gute Institute in diesen Bereichen, Ingenieurswesen, technologische Bereiche, aber das hat nicht geklappt ... Denn du weißt ja, wenn du aus Afrika kommst und auf europäischem Niveau studieren willst ... das ist nicht selbstverständlich! An der Universität, da machst du zwei Jahre, drei Jahre und dann weißt du, gut, ich bin ein Mann, der in einem bestimmten Bereich ausgebildet wurde. Wenn man mich nach meiner Ausbildung fragt, ja, ich habe viele Kurse gemacht: Schweißerei, Industriereinigung, am Hafen, auf den Schiffen, aber eigentlich wollte ich an einem technologischen Institut studieren. ... Wenn du nicht die Möglichkeit hast, an diese kleinen Stellschrauben zu gelangen, an das Wissen ... manchmal im Leben ist es nur eine kleine Information, die alles ändern kann. Eine kleine Orientierung. Manchmal fühle ich mich so, weil ich, besonders ich, ich hatte nie jemanden, der mich angeleitet hat. Ich glaube, dass das vielleicht das Schlimmste für mich war: dieses Fehlen von Hilfe. Wenn ich bloß jemanden zur Orientierung gehabt hätte.

Obwohl Célestin hier deutlich gemacht hat, dass ihm wichtige Informationen und eine Art Vorbild gefehlt hatten, war ich beeindruckt von seinem Streben nach Hoffnung, wie es von Appadurai betont wurde: „Die eigenen Vorstellungskräfte zu mobilisieren, ist besonderes [sic] für die Ärmeren unter den Migranten entscheidend 
wichtig, wenn es um die ,capacity to aspire' geht.“ (Appadurai 2016: 99) Des Weiteren sei es „,nicht ein Privileg von Eliten, Intellektuellen oder kosmopolitischen Geistern ..., sich ihrer Einbildungskraft zu bedienen. Auch die Armen nehmen dieses Recht in Anspruch - nicht zuletzt, indem sie weltweit ihre Migrationsmöglichkeiten nutzen, ob es sie nun in die Nähe oder in die Ferne zieht.“" (ebd.) Und so ließ sich auch Célestin trotz ungewisser Zukunft nicht seiner Hoffnung berauben, als wir uns im Dezember 2016, kurz vor Ende meines letzten längeren Forschungsaufenthaltes getroffen haben.

Die Monate darauf blieben wir in unregelmäßigem Kontakt. Im Mai 2017 schrieb er mir, dass er eine positive Rückmeldung des CNDA erhalten hatte. Fünfzehn Jahre, nachdem er Kamerun verlassen hatte, wurde Célestin Asyl in Französisch-Guyana und folglich in Frankreich und der Europäischen Union gewährt. Im Eingangszitat habe ich Célestins Worte wiedergegeben: „Ich sage mir immer, dass sich eines Tages eine Gelegenheit ergeben wird und vielleicht können sich die Dinge ändern." Er sollte Recht behalten: Im Mai 2017 haben sich die Dinge für Célestin von Grund auf geändert.

\section{Schlussbetrachtung: Von Französisch-Guyana nach ...?}

Célestins Lebensgeschichte zeigt auf erstaunliche Weise die möglichen Verquickungen von Staatsbürgerschaft mit anderen Kategorien sozialer Stratifikation wie Klasse, race und Religion. Da seine Möglichkeiten der sozialen wie geografischen Mobilität qua Geburt extrem eingeschränkt waren, entschied er sich nach einer in Kamerun und der unteren Mittelklasse verbrachten Kindheit und Jugend für eine Migration nach Europa. Obwohl er in Kamerun Bildungserfolge vorweisen konnte, sah er im globalen Norden größere Möglichkeiten, sich beruflich wie persönlich zu entfalten. Nach einer gefährlichen Route durch die Sahara und Nordafrika gelang er schließlich in die spanische Exklave Melilla, von dort aus nach Madrid, Santander und Barcelona, um Spanien schließlich auf dem Höhepunkt der Weltfinanzkrise gemeinsam mit Marcia zu verlassen. Globale Phänomene wie die Weltfinanzkrise oder zuvor schon die Terroranschläge in den Vereinigten Staaten wirkten sich ganz konkret auf Célestins Leben aus und führten zu unvorhergesehenen Entscheidungen. Wie viele andere Menschen weltweit lotete er die sich stets neu ergebenen (Un-)Möglichkeiten der Grenzüberschreitung und Migration je nach Kontext aus. Niemals hätte Célestin bei seiner Abreise aus Kamerun gedacht, eines Tages nach Brasilien auszuwandern. Französisch-Guyana war wie für viele andere Menschen damals noch ein blinder Fleck für ihn. Erst mit der Migration nach Brasilien, wo sich erneut keine Möglichkeiten für ihn und seine Partnerin auftaten, wurde FranzösischGuyana zu einer Art südamerikanischer, kolonial verwobener „Hintertür“ nach Frankreich und in die Europäische Union. Obwohl diese „Hintertür“ seit Jahrzehnten von Tausenden Migrant*innen aus der Region, in den vergangenen Jahren verstärkt auch von Migrant*innen aus Ländern wie Syrien und Palästina genutzt und 
immer stärker durch Grenzkontrollen geschlossen wird (Brancato 2020; Santos im Druck), hat sich die Soziologie bislang kaum mit den 22 in der einen oder anderen Form mit der EU kolonial verstrickten Territorien befasst. ${ }^{12}$ Dies verdeutlicht einmal mehr, dass Migrant*innen die Landkarten in ihrer Not gründlicher studieren als viele in ihrem Eurozentrismus gefangene Soziolog*innen.

Eine Herausforderung biografischer Analysen besteht immer auch in dem Umstand, dass das Leben der interviewten Menschen in aller Regel weitergeht. Célestin und ich stehen seit unserem Kennenlernen in unregelmäßigem Austausch, der sich größtenteils über Instant Messaging abspielt. Zudem hatte ich 2018 und 2019 durch Konferenzreisen nach Cayenne die Möglichkeit, Célestin erneut zu treffen. Zu beiden Zeitpunkten lebte er in Matoury, gleich neben dem Flughafen, von dem er mich 2018 mit seinem Auto abholte. Dem in die Jahre gekommenen Auto fehlten Griffe und etliche technische Details, aber es fuhr und war in Französisch-Guyana, wo es kaum öffentliche Verkehrsmittel gibt, geradezu essentiell. Célestin war müde und euphorisch zugleich, als wir uns wiedersahen. Die Müdigkeit kam, wie er mir sagte, von seinem anstrengenden Job als Gärtnergehilfe, den er von den frühen Morgenstunden bis mittags erledigt. Zu den weiteren Neuigkeiten gehörte auch die Tatsache, dass sein zuvor genannter Wunsch tatsächlich wahr geworden war: Er war mittlerweile an der Université de Guyane immatrikuliert und studierte Informatik. Auch im Privaten hat sich einiges geändert: Célestin war schon seit einiger Zeit nicht mehr mit Marcia zusammen. Mittlerweile lebte er in Matoury mit einer haitianischen Frau zusammen, die ein Kind von ihm erwartete. Wenige Wochen später wurde er Vater eines gesunden Sohnes. Zweifelsohne hat dieser durch seinen Geburtsort ein besseres Los als seine Eltern in der globalen „Geburtsrechtlotterie“ gezogen.

Ende 2019 fand das erneute und vorerst letzte Wiedersehen statt. Ich wartete vor dem Rathaus von Matoury auf Célestin, der kurz nach mir ankam und mich in sein neues Auto winkte. Vorne auf dem Beifahrer*innensitz lag sein Sohn, der kurz zuvor ein Jahr alt geworden war, in einer Babyschale und schaute mich mit großen Augen an. Wir fuhren einige Minuten zu der Wohnung von Célestin und seiner Partnerin. Nachdem Célestins Sohn mein Geburtstagsgeschenk für ihn auspackte und damit spielte, haben Célestin und ich uns über das aktuelle Weltgeschehen, aber auch über unsere privaten und beruflichen Pläne unterhalten. Er hat sich über den $\mathrm{Ab}$ schluss meiner Promotion gefreut und sagte im Anschluss, dass sein Studium pausiere. Neben der Arbeit und seiner neuen familiären Verantwortung bliebe kaum Zeit für ein Studium. Zudem erzählte er mir erneut von einem Plan, den er schon bei unserem Treffen im Jahr zuvor erwähnt hatte. Langfristig, so sagte er mir, wolle er die französische Staatsbürgerschaft beantragen. Interessanterweise stehen aber nicht so sehr das mit der Staatsbürgerschaft verknüpfte, uneingeschränkte Arbeitsund Aufenthaltsrecht im Zentrum seines Interesses, sondern die an die Staatsbürgerschaft gekoppelten, globalen Mobilitätsprivilegien. Seine Zukunft sehe er nämlich

12 Hierzu zählen neben den „Gebieten in äußerster Randlage“ auch die mit ähnlich sperrigem und euphemistischem Titel versehenen „Überseeischen Länder und Hoheitsgebiete“ (Adler-Nissen/Gad 2013), deren Zahl sich durch den Brexit reduziert hat. 
in Kanada. Und so bleibt abzuwarten, wo Célestin - immer noch nach Hoffnung in einem von Ungleichheiten gekennzeichneten Weltsystem strebend - in Zukunft leben wird.

\section{Literatur}

Adler-Nissen, R./Gad, U. P. (2013): European Integration and Postcolonial Sovereignty Games. The EU Overseas Countries and Territories. London: Routledge.

Alpes, M. J. (2017): Why Aspiring Migrants Trust Migration Brokers. The Moral Economy of Departure in Anglophone Cameroon. In: Africa, 87(2), 304-321.

Andersson, R. (2014): Illegality, Inc. Clandestine Migration and the Business of Bordering Europe. Oakland, CA: University of California Press.

Appadurai, A. (2016): Streben nach Hoffnung. Das Narrativ der Flucht und die Ideologie des Nationalstaats. In: Blätter für deutsche und internationale Politik, 1/2016, 95-103.

Arouck, R. de C. (2000): Brasileiros na Guiana francesa. Novas migrações internacionais ou exportação de tensões sociais na Amazônia? In: Lusotopie, 7 , 67-78.

Bahl, E. (2017): Precarious Transnational Biographies. Moroccan Juveniles in the Spanish Enclaves of Ceuta and Melilla. In: Rosenthal, G./Bogner, A. (Hrsg.): Biographies in the Global South. Life Stories Embedded in Figurations and Discourses. Frankfurt a. M./New York: Campus, 185-208.

Boatcă, M. (2015): Global Inequalities Beyond Occidentalism. Farnham: Ashgate.

Boatcă, M. (2018): Caribbean Europe. Out of Sight, Out of Mind? In: Reiter, B. (Hrsg.): Constructing the Pluriverse. The Geopolitics of Knowledge. Durham: Duke University Press, 197-218.

Boatcă, M. (2019): Forgotten Europes. Rethinking Regional Entanglements from the Caribbean. In: Bringel, B./Cairo, H. (Hrsg.): Critical Geopolitics and Regional (Re)Configurations. Interregionalism and Transnationalism between Latin America and Europe. London: Routledge, 96-116.

Brancato, Rémi (2020): L'incroyable périple des Syriens demandeurs d'asile en Guyane. France Inter, 08. Februar 2020. Online: https://www.franceinter.fr/societe/1-incroyable-periple-des-syriensdemandeurs-d-asile-en-guyane $<15.03 .2020>$. 
Braig, M./Costa, S./Göbel, B. (2015): Desigualdades sociales e interdependencias globales en América Latina. Una valoración provisional. In: Revista Mexicana de Ciencias Políticas y Sociales, 223, 209-236.

Crawley, H./Düvell, F./Jones, K./McMahon, S./Sigona, N. (2017): Unravelling Europe's "Migration Crisis". Journeys Over Land and Sea. Bristol: Policy Press.

El País (2017): Especial CIE. Online: https://elpais.com/agr/cies_la_reforma_pendiente/a $<15.03 .2020>$.

European Commission (2011): The Global Approach to Migration and Mobility. Communication from the Commission to the European Parliament, the Council, the European Economic and Social Committee and the Committee of the Regions. Online: https://eur-lex.europa.eu/legal-content/EN/TXT/ PDF/?uri=CELEX:52011DC0743\&from=EN $<15.03 .2020>$.

Hamood, S. (2008): EU-Libya Cooperation on Migration. A Raw Deal for Refugees and Migrants? In: Journal of Refugee Studies, 21(1), 19-42.

Jelin, E./Motta, R./Costa, S. (2018): Global Entangled Inequalities. Conceptual Debates and Evidence from Latin America. London: Routledge.

Korzeniewicz, R. P./Moran, T. P. (2009): Unveiling Inequality. A World-Historical Perspective. London: Russell Sage Foundation.

Kreckel, R. (2008): Soziologie der sozialen Ungleichheit im globalen Kontext. In: Bayer, M./Mordt, G./Terpe, S./Winter, M. (Hrsg.): Transnationale Ungleichheitsforschung. Eine neue Herausforderung für die Soziologie. Frankfurt a. M./New York: Campus, 23-69.

Mam Lam Fouck, S. (2015): La société guyanaise à l'épreuve des migrations du dernier demi-siècle, 1965-2015. Matoury: Ibis Rouge Éditions.

Marshall, B. (2009): The French Atlantic. Travels in Culture and History. Liverpool: Liverpool University Press.

Martins, C. das C./Superti, E./Pinto, M. de J. de S. (2016): Des migrants brésiliens entre le Brésil et la Guyane française. Un espace social en construction ou en tension permanente dans un contexte (trans) frontalier. In: Cahiers internationaux de sociolinguistique, 9(1), 221-242.

Mberu, B. U./Pongou, R. (2016): Crossing Boundaries. Internal, Regional and International Migration in Cameroon. In: International Migration, 54(1), 100118. 
Murdoch, A. (2015): Continental Creolization. French Exclusion through a Glissantian Prism. In: Gutiérrez Rodríguez, E./Tate, S. A. (Hrsg.): Creolizing Europe. Legacies and Transformations. Liverpool: Liverpool University Press, $57-79$.

Piantoni, F. (2011): Migrants en Guyane. Arles/Cayenne: Actes sud/Musée des cultures guyanaises.

Pinto, M. de J. de S. (2016): Migraçao de brasileiros para Guiana Francesa. Da sedução à realidade migratória. In: Collomb, G. /Mam Lam Fouck, S. (Hrsg.): Mobilités, ethnicités, diversité culturelle. La Guyane entre Surinam et Brésil: eléments de compréhension de la situation guyanaise. Matoury: Ibis Rouge Éditions, 173-188.

Police, G. (2010): €udorado. Le discours brésilien sur la Guyane française. Matoury: Ibis Rouge Éditions.

Prates, D. M./Fritz, B./Paula, L. F. de P. (2017): Brazil at Crossroads. A Critical Assessment of Developmentalist Policies. In: Arestis, P./Troncoso Baltar, C./Prates, D. M. (Hrsg.): The Brazilian Economy since the Great Financial Crisis of 2007/2008. Cham: Springer International Publishing, 9-39.

Price, R. (2018): The Oldest Daughter of Overseas France. In: Wood, S./MacLeod, C. (Hrsg.): Locating Guyane. Liverpool: Liverpool University Press, 17-32.

Rosenthal, G. (2016): Die Erforschung kollektiver und individueller Dynamik. Zu einer historisch und prozess-soziologisch orientierten interpretativen Sozialforschung. In: Forum Qualitative Sozialforschung, 17(2), Art. 13. Online: https://www.qualitative-research.net/index.php/fqs/article/view/2614 $<03.09 .2020>$.

Rosenthal, G./Bahl, E./Worm, A. (2017): Illegalized Migration Courses from the Perspective of Biographical Research and Figurational Sociology. The Land Border between Spain and Morocco. In: Rosenthal, G./Bogner, A. (Hrsg): Biographies in the Global South. Life Stories Embedded in Figurations and Discourses. Frankfurt a. M./New York: Campus, 103-159.

Santos, F. (2017): Re-Mapping Europe. Field Notes from the French-Brazilian Borderland. In: InterDisciplines. Journal of History and Sociology, 8(2), 173201.

Santos, F. (2020): Crisscrossing the Oyapock River. Entangled Histories and Fluid Identities in the French-Brazilian Borderland. In: Rein, R./Rinke, S./Sheinin, D. M. K. (Hrsg.): Migrants, Refugees, and Asylum Seekers in Latin America. Leiden: Brill, 217-241. 
Santos, F. (im Druck): Outermost Migrations: Linking French Guiana to the "European Refugee Crisis". In: Boatcă, M. (Hrsg.): De-Linking. Critical Thought and Radical Politics. London: Routledge.

Schieritz, M. (2017): Why Is the World's Poorest Country so Poor? In: Zeit Online, 25. April 2017. Online: https://www.zeit.de/wirtschaft/201704/central-african-republic-poverty-development-assistanceafrica/komplettansicht $<15.03 .2020>$.

Shachar, A. (2009): The Birthright Lottery. Citizenship and Global Inequality. Cambridge, MA: Harvard University Press.

Shachar, A. (2014): Introduction. Citizenship and the 'Right to Have Rights'. In: Citizenship Studies, 18(2), 114-124.

Simon, J. (2006): Irregular Transit Migration in the Mediterranean. Facts, Figures and Insights. In: Sørensen, N. N. (Hrsg.): Mediterranean Transit Migration. Copenhagen: Danish Institute for International Studies, 25-65.

Weiß, A. (2017): Soziologie Globaler Ungleichheiten. Berlin: Suhrkamp. 